\title{
MUDANÇAS DO DESEMPENHO DA FORÇA EXPLOSIVA DURANTE UM CICLO ANUAL EM VOLEIBOLISTAS NA PUBERDADE
}

\author{
Jefferson Eduardo Hespanhol, Pontifícia Universidade Católica de Campinas - PUCC-Campinas - \\ Campinas, São Paulo - Brasil \\ Miguel de Arruda, Faculdade de Educação Física - UNICAMP - Campinas, São Paulo - Brasil
}

\section{RESUMO}

O propósito desse estudo consistiu em investigações nas mudanças no desempenho da força explosiva (FE), força explosiva elástica (FEE), força explosiva elástica reflexa (FEER) em voleibolistas durante um ciclo anual. A amostra examinada foi composta por 43 voleibolistas do sexo masculino. Os desempenhos das variáveis dependentes foram mensurados através dos testes de salto verticais com meio agachamento partindo de uma posição estática (SJ), teste de salto vertical com contra-movimento, sem a contribuição dos membros superiores (CMJ), e o teste de saltos verticais contínuos com duração de 5 segundos sem a contribuição dos joelhos e membros superiores (CJ5s). As coletas foram realizadas antes e depois dos períodos de preparação e competição, sendo as medidas feitas em tapete de contato Jump Test. Superiores desempenhos dos pos-púberes comparado aos púberes foram observados. Em púberes foram observadas significantes mudanças no ciclo anual nas variáveis do desempenho da FE e FEE apresentando aumentos, no entanto não foram encontradas mudanças significantes na FEER, mesmo que os resultados revelando aumentos de desempenho. Os pos-púberes demonstraram aumentos significantes para os desempenhos da FE, FEE e FEER. Deste modo conclui-se que as mudanças foram lineares para os púberes e não lineares para os pós-púberes.

Palavras-Chave: Treinamento (Voleibol); Jovens-Atividades esportivas; Maturação; Puberdade; Força.

\section{CHANGE THE PERFORMANCE IN THE STRENGTH EXPLOSIVE DURING YEAR CYCLE IN YOUNG VOLLEYBALL PLAYER'S IN THE PUBERTY}

\begin{abstract}
The objective of this study it consisted of verify in the changes in the performance of the explosive strength (ES), elastic explosive strength (EES), and reflex explosive strength elastic (REES) in volleyballs players during a years cycle. The sample was composed for 45 volleyball players of the man sex. The participants were submitted the anthropometrical measures (stature, body weight, percentage of fat, fat mass, lean body mass, and thigh muscle area), and vertical jump tests (squat jump, countermovement jump, and continuous jumps $5 \mathrm{~s}$ ) at three moments: in the beginning and the end of the pre-season; in the beginning and the end of the season, being the measures made in carpet of contact Jump Test. Higher performances of the after-pubescent than pubescent were observed. In pubescent significant changes in the annual cycle in the variable of the performance of ES and EES had been observed presenting
\end{abstract}


increases, however, the changes had not been significant in the REES, even if showed increases the performance. The after-pubescent showed significant increases in performances the ES, EES, and. In this way one concludes that the changes had been linear for pubescent and the not linear ones for the after-pubescent.

KEY-WORDS: Training (Volleyball); Young-Sports activity; Maturation; Puberty; Strength. 


\section{INTRODUÇÃO}

Treinabilidade denota o grau de adaptabilidade e de modificação positiva do estado funcional e morfológico dos praticantes, ${ }^{1}$ que denotam o grau de sensibilidade do sistema corporal, do organismo, tecidos e componentes físicos em relação a um programa de treinamento, os quais recebem influencia da especificidade dos estímulos de exercícios.

A questão que desafia a treinabilidade em seu contexto específico de modificação positiva é saber se o crescimento e a maturação são responsáveis pelo desenvolvimento especifico ou se o estímulo de treinamento é o responsável por tais mudanças.

O interesse científico por parte dos pesquisadores, pelo monitoramento dos fenômenos crescimento, desenvolvimento e maturação, tem sua gênese na convicção da necessidade de estudar a treinabilidade, com a finalidade de verificar a sensibilidade e especificidade das respostas dos estímulos de treinamento. ${ }^{2}$

A propósito, no que se refere ao fator treinabilidade, é cada vez mais evidente sua importância em relação ao crescimento, maturação, desenvolvimento e desempenho competitivo na infância e na adolescência. Nesse aspecto entende-se que a utilidade das informações produzidas por esse monitoramento esteja na atuação dos profissionais de EDUCAÇÃO FÍSICA e da área de saúde, na construção de programas de intervenção, no caso de programas de treinamento em jovens atletas.

Haja vista a necessidade de se levar em consideração na construção desses programas as variações no crescimento, na maturação, no desenvolvimento e no desempenho competitivo provocadas pela sensibilidade e especificidade dos estímulos do treinamento em crianças e adolescentes.

As várias expressões das diferentes funções biológicas não atingem plena funcionalidade, eficiência e eficácia antes de se atingir a maturidade biológica. Sendo assim, vários estudos ${ }^{2-4}$ consideram que existem aumentos no desenvolvimento da força até aos 20 anos de idade relacionados a maturidade.

Conexões: revista da Faculdade de Educação Física da UNICAMP, Campinas, v. 8, n. 3, p. 64-83, set./dez. 2010.

ISSN: 1983-9030 
Essas observações vislumbram a existência da variação da maturação sobre o desenvolvimento da força durante a puberdade. Na treinabilidade da força, estudos consideram efetivos aumentos do desempenho da força máxima ${ }^{5-11}$ representadas pelo aumento de uma repetição máxima em jovens atletas. Nas investigações sobre a treinabilidade da força explosiva, comprovadamente, as informações ao se empregar a técnica de salto vertical squat jump foram notadas aumentos no desenvolvimento de força. ${ }^{9,12-13}$ Com outra manifestação da força explosiva elástica, representada pela técnica de salto com contra movimento, observaram aumentos na altura saltada, ${ }^{9-14}$ considerando aumentos no desenvolvimento da força.

Todavia, não existe um consenso de que o status da maturidade e o treinamento da força sejam fatores relevantes para o desenvolvimento da força durante o ciclo anual e o planejamento de vários anos. Para alguns estudiosos, ${ }^{15,2}$ o tempo de treinamento e o status maturacional têm influência no aumento do desempenho das manifestações da força, porém em atletas de alto rendimento o aumento da força é mais limitado do que em jovens atletas, tornando difícil a tarefa de determinar com precisão e consistência o tipo, a carga, a densidade e o volume de treinamento prescrito como ótimo. Para cada um dos indivíduos de diversas categorias, idades cronológicas e níveis maturacionais são determinados diferentes mudanças durante a preparação e competição em jovens atletas. ${ }^{16}$

Nota-se que o treinamento específico de força, o desenvolvimento da força e a idade biológica têm efeitos nas mudanças do desempenho físico na puberdade. ${ }^{3,17-19}$ Contudo, há uma falha na compreensão da literatura sobre as mudanças da força explosiva em jovens atletas voleibolistas na puberdade, diante desse problema se faz presente uma questão: será que constitui a existência de mudanças diferentes do desenvolvimento da força explosiva durante um ciclo anual em voleibolistas na puberdade.

Diante disso, o objetivo desse estudo foi investigar as mudanças no desempenho da força explosiva, força explosiva elástica e força explosiva elástica reflexa em voleibolistas do sexo masculino durante os períodos de preparação, competição e ciclo anual. 


\section{MÉTODO}

Sujeitos: Os participantes desse estudo foram 43 voleibolistas, na faixa etária de 14 a 19 anos pertencente a as categorias infantil, infanto-juvenil e juvenil de uma equipe da região metropolitana de Campinas - São Paulo; foram divididos em dois grupos púberes ( $\mathrm{n}=10$; $16,15 \pm 1,75 \mathrm{anos}, 185,56 \pm 6,25 \mathrm{~cm})$ e pós-púberes ( $\mathrm{n}=33 ; 17,68 \pm 1,42 \mathrm{anos}, 190,85 \pm 7,13 \mathrm{~cm})$ conforme procedimento de classificação maturacional, ressalta ques não foram encontrados nessa amostragem sujeitos pré-puberes. O projeto foi aprovado pelo Comitê de Ética em Pesquisa da Universidade Estadual de Campinas (UNICAMP).

Delineamento do estudo: Estudo longitudinal de investigação das mudanças no desempenho da FE, FEE e FEER em voleibolistas do sexo masculino nos períodos de preparação, competição e ciclo anual. Na realização dos testes 1 e 2, foi possível observar as mudanças ocorridas após o período de preparação, (16 semanas); As mudanças ocorridas no período de competição, na comparação dos testes 2 e 3, (26 semanas), e na comparação dos testes 1 e 3 foi possível observar as mudanças no ciclo anual, (42 semanas).

Variáveis do estudo: As variáveis desse estudo foram compreendidas no desempenho da força, correspondendo à força explosiva (FE), força explosiva elástica (FEE) e força explosiva elástica (FEER), as medidas antropométricas e maturação biológica.

Medidas antropométricas: Para as variáveis antropométricas foram observadas através das verificações das seguintes medidas: estatura, massa corporal, circunferências da coxa e dobras cutâneas. A Área Muscular da coxa foi estimada a partir da equação descrita por Frisancho. ${ }^{20}$

Avaliação maturacional: A variável da maturação biológica foi observada através da avaliação do estágio maturacional, cuja determinação das medidas do desenvolvimento de caracteres sexuais secundários, seguiu-se o procedimento descrito por Tanner, ${ }^{21}$ neste procedimento foi utilizado uma prancha com fotografias dos diferentes estágios (1, 2, 3, 4 e 5) de desenvolvimento para dos pêlos pubianos e os genitais propostos para o sexo masculino. A classificação dos sujeitos em púberes e pós-púberes foram estabelecidas seguindo a padronização descrita por Tanner, ${ }^{21}$ sendo estágios de genitália e pêlos pubianos dois, três e 
quatro foram classificados como púberes, para os pós-púberes o estágio foi o cinco para ambas as características.

Procedimentos dos Testes: Para medir a expressão da força explosiva, o pesquisador empregou a técnica de salto vertical com meio agachamento partindo de uma posição estática sem contra movimento $(\boldsymbol{S} \boldsymbol{J})$, Para a expressão da força explosiva elástica, foi empregada a técnica de salto vertical com contra movimento sem a utilização dos membros superiores $(\boldsymbol{C M J})$. Quanto a expressão da FEER foi utilizado o teste de saltos verticais contínuos com duração de 5 segundos sem a contribuição dos joelhos e membros superiores (CJ5s). O teste de CJ5s foi realizado com o procedimento descrito por Bosco et al.; ${ }^{22} \boldsymbol{S J}$ e $\boldsymbol{C M J}$ foi utilizado a técnica descrita por Komi; Bosco. ${ }^{23} \mathrm{O}$ resultado expresso pelo rendimento do impulso na vertical indicou a altura saltada em centímetros.

Equipamento: Para a coleta de dados nos testes de saltos $\boldsymbol{S J}, \boldsymbol{C M J}$ e $\boldsymbol{C J 5 s}$ foi utilizado o tapete de contacto JUMP TEST, que mede $40 \mathrm{~cm}$ de largura por $80 \mathrm{~cm}$ de comprimento, pesa em torno de 2,3 kg, e tem um cabo para conexão a um computador Pentium IV $1.4 \mathrm{GHz}$. O aparelho informa medidas sobre altura do salto $(\mathrm{cm})$, velocidade do movimento, tempo de vôo (m.sec.) e contato (m.sec.), incluindo a somatória da altura dos saltada (cm) e número de saltos verticais $\left(\mathrm{n}^{\circ}\right)$. É registrado o tempo de vôo durante o salto, sendo a altura atingida pelo centro de gravidade, isto é, a altura do salto.

Qualidade das medidas: O coeficiente de correlação intraclasse foi calculado para cada variável dos testes, demonstrando níveis altos de confiabilidade $(\boldsymbol{C M J}=0,98 ; \boldsymbol{S J}=0,99 ; \boldsymbol{C J} \boldsymbol{s}$ $=0,91)$ no teste/reteste com saltos verticais, apresentando coeficientes de variações baixos $(\mathrm{CV}=2,34 \% ; 2,68 \% ; 3,41 \%$, respectivamente para $\boldsymbol{S J}, \boldsymbol{C M J}$ e $\boldsymbol{C J 5 s})$.

Coleta de dados: Houve um processo de familiarização para os testes de saltos verticais, isto antes da coleta definitiva do teste 1. A coleta de dados para os testes 1, 2 e 3, foram desenvolvidas seguindo a ordenação do primeiro dia de coleta em que se desenvolveu a aplicação das medições da antropometria e maturação biológica e do segundo dia em que empregou-se a medição dos testes físicos do salto vertical: $\boldsymbol{S J}, \boldsymbol{C M J}, \boldsymbol{C J 5 s}$. Para os testes de saltos verticais, os atletas executaram um aquecimento de 10 minutos contendo exercícios de 
alongamento, corridas, coordenativos, e de saltos verticais com as respectivas técnicas. A ordenação de execução dos testes físicos foram o teste de salto vertical $\boldsymbol{S J}, \boldsymbol{C M J}$, e $\boldsymbol{C J 5}$ s. O intervalo entre os testes foi de dois (2) minutos. Para a execução dos testes foi seguindo o descrito nos procedimentos técnicos, com a meta de saltar o máximo possível durante todos os saltos verticais. Cada sujeito realizou três tentativas máximas para cada um das condições dos três testes com saltos verticais contendo suficiente recuperação entre as tentativas (10 segundos). O melhor das tentativas de cada técnica de salto vertical foi escolhido como o escore de medida para a análise.

Procedimento de analise estatística: Inicialmente foram empregadas as técnicas estatísticas descritivas da média, desvio padrão e o delta percentual da magnitude das diferenças para descrição dos resultados e caracterização das variáveis estudadas. Foi empregada a técnica estatística K-S para a verificação da distribuição de normalidade dos dados coletados. Os desempenhos foram comparados através da ANOVA (one way), para as comparações múltiplas utilizou o teste de Bonferoni, sendo que o nível de significância adotado foi de $\mathrm{p}<0,05$.

\section{RESULTADOS}

As mudanças do desempenho da força em voleibolistas púberes e pós-puberes, são apresentados nas Tabelas 1, 23 e 4 .

\section{Comparação das mudanças do desempenho da força em voleibolistas púberes}

\section{Ciclo anual em púberes:}

Significantes mudanças nas variáveis do desempenho da força explosiva (FE) e explosiva

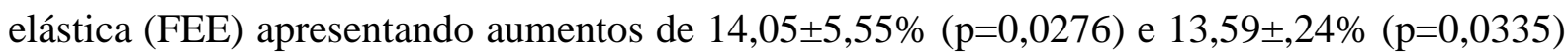
respectivamente, no entanto não foram encontradas mudanças significantes na força explosiva elástica reflexa (FEER), mesmo que os resultados revelando aumentos de desempenho de $13,00 \pm 8,62 \%$.

Conexões: revista da Faculdade de Educação Física da UNICAMP, Campinas, v. 8, n. 3, p. 64-83, set./dez. 2010. ISSN: 1983-9030 
Os índices de elasticidade (IE) e reatividade (IR) demonstraram comportamentos diferenciados após o ciclo anual, quanto ao IE nota-se uma diminuição do índice e uma grande variabilidade de mudanças, as quais não foram estatisticamente significantes para esse estudo em púberes, por outro índice IR observaram aumentos dos resultados e também uma alta variação das mudanças, as quais não foram significantes. Nos indicadores de composição

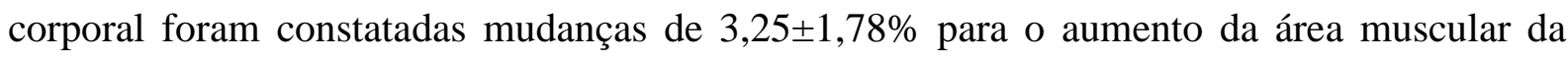

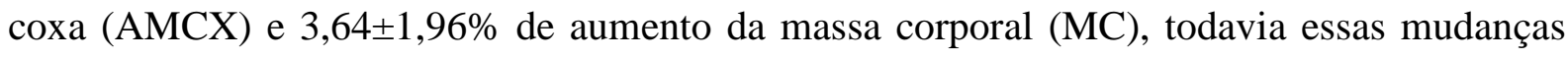
não foram estatisticamente significantes.

\section{Preparação em púberes:}

Na comparação das mudanças ocorridas após a preparação nos indicadores de composição corporal e no desempenho das manifestações de força dos voleibolistas púberes foi possível verificar que não houve nenhuma mudança significante em qualquer das variáveis estudadas, mesmo que aumentos de $8,86 \pm 4,46 \%, 8,45 \pm 5,51 \%$ e $9,98 \pm 5,34 \%$ foram observados nos desempenhos da FE, FEE e FEER, respectivamente. Na AMCX e na MC foram observadas mudanças de $2,49 \pm 1,56 \%$ e de $2,03 \pm 1,61 \%$ respectivamente, no entanto não foram significantes. Os índices demonstraram diminuições de 7,83\% para o IE, e aumentos de $14,62 \pm 16,24 \%$ para o índice de reatividade, as quais não foram significantes.

Tabela 1: Magnitude das mudanças da composição corporal e desempenho das manifestações da força dos voleibolistas púberes após a preparação, competição e ciclo anual

\begin{tabular}{lccccccc}
\hline \multirow{2}{*}{ Variáveis } & \multicolumn{2}{c}{ Preparação } & \multicolumn{2}{c}{ Competição } & \multicolumn{2}{c}{ Ciclo Anual } & \multirow{2}{*}{ p } \\
\cline { 2 - 6 } & Média & $\mathbf{d p}$ & Média & $\mathbf{d p}$ & Média & $\mathbf{d p}$ & \\
\hline AMCX $\left(\mathrm{cm}^{2}\right)$ & 2,49 & 1,56 & 0,74 & 0,32 & 3,25 & 1,78 & 0,6851 \\
MC $(\mathrm{kg})$ & 2,03 & 1,61 & 1,54 & 1,61 & 3,64 & 1,96 & 0,8940 \\
FE $(\mathrm{cm})$ & 8,86 & 4,46 & 4,77 & 2,62 & $14,05^{*}$ & 5,55 & $\mathbf{0 , 0 2 7 6}$ \\
FEE $(\mathrm{cm})$ & 8,45 & 5,51 & 4,73 & 1,63 & $13,59^{*}$ & 6,24 & $\mathbf{0 , 0 3 3 5}$ \\
FEER $(\mathrm{cm})$ & 9,88 & 5,34 & 2,79 & 4,68 & 13,00 & 8,62 & 0,0557 \\
IE $(\%)$ & $-7,83$ & 38,62 & $-5,29$ & 38,11 & $-17,56$ & 81,68 & 0,7063 \\
IR $(\%)$ & 14,62 & 16,24 & 1,01 & 3,68 & 16,15 & 19,91 & 0,1063 \\
\hline
\end{tabular}

MCM=Massa Corporal Magra; AMCX=Área Muscular da Coxa; FE=Força Explosiva; FEE=Força Explosiva Elástica; FEER=Força Explosiva Elástica Reflexa; IE=Índice de Elasticidade; IR=Índice de Reatividade; ; p $<0,05$ quanto o teste 1 para 3. 


\section{Competição em púberes:}

As mudanças ocorridas no desenvolvimento do período de competição foram sumarizadas na Tabela 3. Não foram percebidas mudanças estatisticamente significantes em nenhuma das variáveis estudadas. Todavia notam-se aumentos de desempenho das manifestações da força quanto a FE $(4,77 \pm 2,62 \%)$, a FEE $(4,73 \pm 1,63 \%)$ e para FEER $(2,79 \pm 4,68 \%)$. Na AMCX e na MC foram observadas mudanças de $0,74 \pm 0,32 \%$ e de $1,54 \pm 1,61 \%$ respectivamente. Os índices demonstraram diminuições de $5,29 \pm 38,11 \%$ para o IE, e aumentos de $1,01 \pm 3,68 \%$ para o de reatividade.

Tabela 2: Demonstrativo dos indicadores de composição corporal e desempenho das manifestações da força dos voleibolistas púberes nos respectivos testes

\begin{tabular}{lcccccc}
\hline \multirow{2}{*}{ Variáveis } & \multicolumn{2}{c}{ Teste 1 } & \multicolumn{2}{c}{ Teste 2 } & \multicolumn{2}{c}{ Teste 3 } \\
\cline { 2 - 7 } & Média & dp & Média & dp & Média & dp \\
\hline AMCX $\left(\mathrm{cm}^{2}\right)$ & 190,72 & 29,12 & 195,27 & 28,41 & 196,67 & 28,42 \\
MC $(\mathrm{kg})$ & 70,44 & 9,49 & 71,90 & 10,47 & 72,93 & 9,78 \\
FE $(\mathrm{cm})$ & 31,49 & 3,72 & 34,16 & 3,06 & 35,79 & 3,26 \\
FEE $(\mathrm{cm})$ & 34,46 & 4,31 & 37,24 & 3,61 & 38,95 & 3,22 \\
FEER(cm) & 35,66 & 4,89 & 39,03 & 4,21 & 39,99 & 3,23 \\
IE $(\%)$ & 9,45 & 3,79 & 8,95 & 1,54 & 8,92 & 1,97 \\
IR (\%) & 2,80 & 0,55 & 3,13 & 0,27 & 3,16 & 0,22 \\
\hline
\end{tabular}

MCM=Massa Corporal Magra; AMCX=Área Muscular da Coxa; FE=Força Explosiva; FEE=Força Explosiva Elástica; FEER=Força Explosiva Elástica Reflexa; IE=Índice de Elasticidade; IR=Índice de Reatividade.

\section{Comparação das mudanças do desempenho da força em voleibolistas pos-púberes}

\section{Ciclo anual em pos-púberes:}

No ciclo anual (Tabela 3 e 4) demonstraram aumentos significantes de 12,91 $\pm 5,46 \%$ $(\mathrm{p}<0,001), 10,11 \pm 3,72 \% \quad(\mathrm{p}<0,001)$ e $13,07 \pm 6,82 \% \quad(\mathrm{p}<0,001)$ respectivamente para os desempenhos da FE, FEE e FEER. Nos índices de reatividade consta-se aumentos

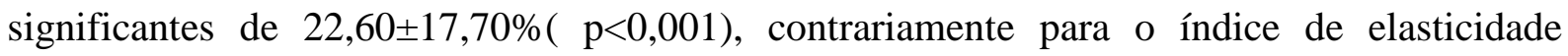
observaram diminuições significantes de $-9,93 \pm 42,06 \%(\mathrm{p}=0,0180)$. Entretanto, não foram constatados aumentos significantes nos indicadores de composição corporal, os quais

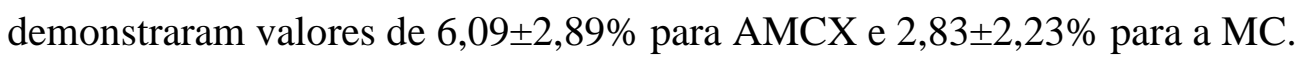




\section{Preparação em pos-púberes:}

Aumentos significantes foram encontrados no desempenho da FE, FEE e FEER, representando valores de $10,58 \pm 5,72 \%$ ( $p=0,0001), 8,46 \pm 4,38 \%(p=0,0024)$ e $8,62 \pm 6,21 \%$ $(\mathrm{p}=0,0022)$, respectivamente. Conseqüentemente, na comparação dos índices foram percebidos aumentos significantes no IR, o qual representou mudanças de $15,87 \pm 16,28 \%$ $(\mathrm{p}=0,0017)$, no outro IE foram observadas diminuições significantes de $-6,70 \pm 6,21 \%$ $(\mathrm{p}=0,0018)$. Embora observados aumentos na AMCX de 3,09 $\pm 2,49 \%$ e para MC de $2,67 \pm 2,46 \%$ para os voleibolistas pos-púberes após a preparação.

Tabela 3: Magnitude das mudanças da composição corporal e desempenho das manifestações da força dos voleibolistas pos-púberes após a preparação, competição e ciclo anual

\begin{tabular}{lccccccc}
\hline \multirow{2}{*}{ Variáveis } & \multicolumn{2}{c}{ Preparação } & \multicolumn{2}{c}{ Competição } & \multicolumn{2}{c}{ Ciclo Anual } & \multirow{2}{*}{ p } \\
\cline { 2 - 6 } & Média & dp & Média & dp & Média & dp & \\
\hline AMCX $\left(\mathrm{cm}^{2}\right)$ & 3,09 & 2,49 & 2,92 & 2,08 & 6,09 & 2,89 & 0,2183 \\
MC $(\mathrm{kg})$ & 2,67 & 2,46 & 0,19 & 0,99 & 2,83 & 2,23 & 0,3411 \\
FE $(\mathrm{cm})$ & $10,58^{\ddagger}$ & 5,72 & 2,48 & 3,95 & $12,91^{*}$ & 5,46 & $\mathrm{p}<0,001$ \\
FEE $(\mathrm{cm})$ & $8,46^{\ddagger}$ & 4,38 & 1,58 & 2,72 & $10,11^{*}$ & 3,72 & $\mathrm{p}<0,001$ \\
FEER(cm) & $8,62^{\ddagger}$ & 6,21 & 4,14 & 3,53 & $13,07^{*}$ & 6,82 & $\mathrm{p}<0,001$ \\
IE $(\%)$ & $-6,70^{\ddagger}$ & 6,21 & $-6,93$ & 21,71 & $-9,93^{*}$ & 42,06 & 0,0180 \\
IR $(\%)$ & $15,87^{\ddagger}$ & 16,28 & $6,81^{\dagger}$ & 4,86 & $22,60^{*}$ & 17,70 & $\mathrm{p}<0,001$ \\
\hline
\end{tabular}

MCM=Massa Corporal Magra; AMCX=Área Muscular da Coxa; FE=Força Explosiva; FEE=Força Explosiva Elástica; FEER=Força Explosiva Elástica Reflexa; IE=Índice de Elasticidade; IR=Índice de Reatividade; " $\mathrm{p}<0,05$ quanto o teste 1 para $3 ;{ }^{\dagger} \mathrm{p}<0,05$ quanto o teste 2 para $3 ;{ }^{*} \mathrm{p}<0,05$ quanto o teste 1 para 2.

\section{Competição em pos-púberes:}

Após a competição em voleibolistas pos-púberes, foram averiguados aumentos

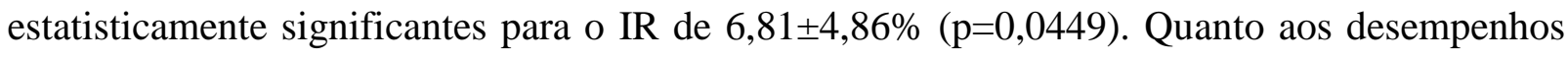
da FE, FEE e FEER não houve aumentos significantes, apenas consideradas mudanças de $2,48 \pm 3,95 \% ; 1,58 \pm 2,72 \%$ e $4,14 \pm 3,53 \%$ após o desenvolvimento do período de competição. Houve aumentos no do IE de $-6,93 \pm 21,71 \%$, mas não significantes. Na AMCX e na MC foram observadas mudanças de $2,92 \pm 2,28 \%$ e de $0,19 \pm 0,99 \%$ respectivamente. 
Tabela 4: Demonstrativo dos indicadores de composição corporal e desempenho das manifestações da força dos voleibolistas pós-púberes nas respectivas avaliações

\begin{tabular}{lcccccc}
\hline \multirow{2}{*}{ Variáveis } & \multicolumn{2}{c}{ Teste 1 } & \multicolumn{2}{c}{ Teste 2 } & \multicolumn{2}{c}{ Teste 3 } \\
\cline { 2 - 7 } & Média & dp & Média & dp & Média & dp \\
\hline AMCX $\left(\mathrm{cm}^{2}\right)$ & 220,08 & 38,46 & 227,26 & 43,10 & 233,41 & 41,03 \\
MC $(\mathrm{kg})$ & 78,13 & 9,07 & 80,20 & 9,23 & 80,32 & 9,08 \\
FE $(\mathrm{cm})$ & 36,97 & 3,10 & 40,74 & 3,73 & 41,66 & 3,04 \\
FEE $(\mathrm{cm})$ & 41,35 & 3,61 & 45,38 & 3,61 & 46,03 & 3,60 \\
FEER(cm) & 42,08 & 4,18 & 45,60 & 4,32 & 47,42 & 3,98 \\
IE $(\%)$ & 13,28 & 4,99 & 11,42 & 3,37 & 10,48 & 3,51 \\
IR $(\%)$ & 3,05 & 0,50 & 3,47 & 0,41 & 3,88 & 0,45 \\
\hline
\end{tabular}

MCM=Massa Corporal Magra; AMCX=Área Muscular da Coxa; FE=Força Explosiva; FEE=Força Explosiva Elástica; FEER=Força Explosiva Elástica Reflexa; IE=Índice de Elasticidade; IR=Índice de Reatividade.

\section{DISCUSSÃO DOS RESULTADOS}

\section{Mudanças em voleibolistas púberes}

Sob o ponto de vista do treinamento, é interessante o desenvolvimento de desempenho das manifestações da força em púberes representadas pelas técnicas de saltos verticais. Considerando a força explosiva estimada pelo teste $\boldsymbol{S J}$, a força explosiva elástica pelo $\boldsymbol{C M J}$, e a força explosiva elástica reflexa representada pelo $\boldsymbol{C j 5 s}$, considerando que todas apresentaram aumentos visíveis de desempenho ao final do ciclo anual do período do teste. Da mesma maneira são visíveis os resultados relativos aos períodos específicos de preparação e competição, os quais não apresentaram aumentos significativos, mas indicações de que em parte no período de preparação houve mudanças, porém sem representação significância na FE, FEE, FEER, e AMCX. Por conseguinte, com o estimulo competitivo continuaram a produzir aumentos nas mudanças de seus desempenhos, mas sem representação significante na FE, FEE, FEER, e AMCX.

Os aumentos de desempenho da FE, apesar de significantes, não foram superiores aos das outras manifestações; esse aumento provavelmente deve ter causas de mudanças relacionadas ao componente neural, dentre as quais são sugeridas modificações no recrutamento das unidades motoras, ${ }^{24} \mathrm{e}$ também às mudanças da sincronização. ${ }^{3}$

Conexões: revista da Faculdade de Educação Física da UNICAMP, Campinas, v. 8, n. 3, p. 64-83, set./dez. 2010. ISSN: 1983-9030 
Alguns estudos apontam para aumentos da força durante a puberdade causada pela hipertrofia muscular. ${ }^{25-27}$ No entanto com certa cautela, os resultados sugerem que não houve aumentos significantes na AMCX, logo, isso não explica os desenvolvimentos do desempenho da força explosiva em púberes, que possa ser causado pelo aumento do componente contrátil.

Por outro lado, em alguns estudos da literatura é observado que uma das causas do aumento da força explosiva se explica pelo aumento do componente neural, representado pelo aumento da força máxima, ${ }^{3,7-8,13,28}$ os quais demonstram que esse regime de treinamento para força máxima em púberes apresentou modificações positivas, sugerindo treinabilidade em jovens desse nível maturacional.

Corroborando com esse fundamento, ${ }^{10}$ evidenciam mudanças na força máxima significantes para ambos os grupos que experimentaram um programa de treinamento como o grupo Controle, no entanto, indicam que o grupo experimental que realizou o programa demonstrou aumentos nos diferentes exercícios de 24,3 a $71,4 \%$, enquanto que no grupo Controle, que indicou o desenvolvimento relativo ao crescimento e maturação, observou-se mudanças de 0 a 4,4\% nos diferentes exercícios. Aqui a uma tendência do fator interveniente seja o treinamento intenso na sua manifestação específica, principalmente, com o treinamento que estimula o componente de recrutamento em púberes. ${ }^{29}$

Sendo assim, na FE percebe-se que tanto os voleibolistas com maiores treinabilidades, quanto os de menores treinabilidades apresentaram mudanças de desempenho da força durante o processo de desenvolvimento de um ciclo anual de treinamento. Ressalta-se que as justificativas relativas a essas mudanças da força explosiva não se dão pelo aumento, somente do recrutamento das unidades motoras, mas também pelo aumento da velocidade de contração. Logo, essas informações emergem uma das limitações desse presente estudo; há ausência de investigações dos indicadores biológicos para sustentar tais afirmações.

É importante a questão relativa às respostas sobre o efeito de treinamento do componente elástico, representada pela FEE, estimada pela técnica de salto vertical CMJ, no qual ficou evidente que os voleibolistas púberes de ambos estados de treinamento receberam influência

Conexões: revista da Faculdade de Educação Física da UNICAMP, Campinas, v. 8, n. 3, p. 64-83, set./dez. 2010.

ISSN: 1983-9030 
do desenvolvimento da força ao longo do ciclo anual. O componente elástico neste estudo apresentou diminuições de $17,56 \%(9,45 \pm 3,79 \%$ para $8,92 \pm 1,97 \%)$, o que demonstrou ser influenciado pelo aumento da FE. Embora o aumento observado na FEE possa ser sugerido pelo aumento dos componentes pertencentes à estrutura da força explosiva representada pelo $\boldsymbol{S J}$, pois seu grau de determinação contribuiu na explicação do $\boldsymbol{C M J}$, a qual foi de $81 \%$. No componente elástico reflexo, os resultados sugerem aumentos expressivos de 13,00\% entretanto, não significantes, representando 2,80 para 3,16\% no índice de reatividade.

Percebeu-se que em atletas púberes, mais treinados no componente elástico reflexo, a tendência é não ter alterações após o ciclo anual final, pois o estimulo realizado no período de competição não é suficiente para apresentar mudanças nesses atletas; isso demonstra certa influência do período de treinamento. Bojsen-Møller et al., ${ }^{30}$ em atleta de alto nível, observaram correlação entre a força máxima, a stiffness no $\boldsymbol{S J}$ e $\boldsymbol{C M J}$ (r = 0.64, p < 0.05 e r = $0.55, \mathrm{p}<0.05)$.

Diante dessas premissas, percebeu-se que os desenvolvimentos da força explosiva em voleibolistas púberes apresentaram certa tendência de maiores sensibilidades dos componentes de recrutamento $^{29}$ indicados pelo SJ, e pelas informações relevantes da literatura ao apontar a força máxima ${ }^{30-31,10}$ como outro elemento de importante colaboração para as modificações positivas desse desenvolvimento, no entanto, esse estudo não investigou essa variável. Em suma, nos fatores anteriormente expostos, o fator genético deve ser considerado nas diferenças entre os jovens atletas nas mudanças ocorridas durante o ciclo anual.

\section{Mudanças em voleibolistas pós-púberes}

O desenvolvimento de desempenho das manifestações da força em pós-púberes apresentou aumentos significantes de FE, FEE e FEER após o ciclo anual, entretanto, o período de maiores mudanças significantes observadas foi o de preparação.

No componente contrátil perceberam-se mudanças lineares durante o ciclo, indicadas pelos aumentos da AMCX para o ciclo anual, sendo similares para a preparação e a competição. Isto sugere certa influência maturacional e não de treinamento específico em pós-púberes voleibolistas.Ficou evidente que os praticantes pós-púberes apresentaram sensibilidade dentro

Conexões: revista da Faculdade de Educação Física da UNICAMP, Campinas, v. 8, n. 3, p. 64-83, set./dez. 2010.

ISSN: 1983-9030 
de todos os componentes estruturais da produção de força, principalmente, se comparados aos períodos de preparação e competição.

A sensibilidade de mudanças nos outros componentes é também observada pela especificidade do programa de preparação e competição, bem como pelas influências maturacionais. Dentro da FE, FEE, FEER com o estímulo do treinamento todos aumentaram seus desempenhos, o que não aconteceu com o estimulo da competição.

Como sustentação dessa afirmação, observa-se em Coutts, Murphy e Dascombe ${ }^{32}$ estudo com jovens atletas de rúgbi, indicadores de que após um programa de exercício de força, houve aumentos significantes na força máxima, $\boldsymbol{S J}$ e $\boldsymbol{C M J}$, após 12 semanas de treinamento do que o grupo de jovens sem treinamento, o que sugere certa tendência de treinabilidade das manifestações de treinamento intenso como finalidades pertinentes a preparação desses jovens. No componente elásticos, o estudo de Gorostiaga et al., ${ }^{33}$ verificou efeitos de treinamento da força com cargas altas em jovens de idade pós pubertária em relação a força explosiva elástica, essa representada pelo $\boldsymbol{C M J}$. Constatou-se que no grupo de treinamento experimental de 11 semanas resultou em aumentos significativos de 5-14\% em CMJ ( $\mathrm{p}<$ 0.01 ), enquanto não houve mudanças no grupo controle.

Diante da descoberta de Nuzzo et al. ${ }^{34}$ cujo relacionamento da força máxima com o $\boldsymbol{C M J}$, reportam aos resultados que sugerem que aumentar da força máxima pode melhorar o desempenho em movimentos explosivos, surge uma outra dúvida sobre as transferências das modificações positivas entre os componentes estruturais; nesse caso houve explicação da intervenção da força máxima no componente elástico da produção de força representada pelo $\boldsymbol{C M J}$. Kubo et al. ${ }^{35}$ com adultos realizando programas de treinamento divididos em grupo prescrições de treinamento de força máxima (TFM, carga 80\% 1RM) e grupo de treinamento de pliometria (TP; hopping e drop jump/DJ usando $40 \%$ de $1 \mathrm{RM}$ ), investigou as mudanças da força explosiva nas técnicas de saltos verticais $\boldsymbol{S J}, \boldsymbol{C M J}$ e $\boldsymbol{D J}$. Com o TP verificou-se aumentos significantes no desempenho de $\boldsymbol{S J}, \boldsymbol{C M J}$, e $\boldsymbol{D J}$, no entanto, com o treino de força máxima aumentou somente $\boldsymbol{S} \boldsymbol{J}$. Entretanto, houve nenhuma diferença significativa entre TFM e TP nas mudanças nas atividades eletromiográfica dos músculos medidos durante saltar. Os resultados indicam que os ganhos do desempenho do salto após o treinamento de pliometria 
estão atribuídos às mudanças nas propriedades mecânicas do complexo do músculo-tendão, melhor que às estratégias da ativação do músculo.

Para os pos-púberes a especificidade do treinamento é um fator a ser considerado nas respostas sobre a sensibilidade de mudanças dos jovens nessa fase de maturidade biológica, visto que, estes respondem com modificações positivas em períodos de preparação, no entanto sobre a especificidade, faltaria para esse estudo controlar as variáveis biológicas que causam as mudanças em seus aspectos funcionais e morfológicos.

Diante desse ponto, torna-se possível realizar considerações relativas às mudanças observadas em voleibolistas pós-púberes, logo, um aspecto considerável é que as maiores magnitudes de mudanças acontecerão após o período de preparação. Deste modo, essas mudanças apresentam caráter de especificidades de treinamento ${ }^{35-37}$ com certa sensibilidade de desenvolvimento em alguns componentes. ${ }^{32-34}$ A grande dificuldade desta análise de mudanças do desempenho foi, sem dúvidas, a falta de estudos para auxiliar na interpretação dos resultados, e também pelas limitações em aprofundar as investigações nas análises dos indicadores biológicos e nas respostas relativas à explicação de componente estrutural de produção de força em pós-púberes.

\section{CONCLUSÃO}

Nos voleibolistas púberes houve uma tendência de mudanças significantes na manifestação da FE e FEE, porém, considerando a analise do IE, existem indícios que o ajuste da treinabilidade tenha ocorrido pelo componente neural da força explosiva. Na sensibilidade das mudanças do desempenho houve indicadores de que os aumentos nos teste para FE e FEE foram mais significante do que FEER e AMCX.

Para os púberes houve aumentos significantes na FE, FEE e FEER após o ciclo anual, sendo significantes no período de preparação, mas apenas expressivos após a competição, no entanto, após a competição, a tendência foi aumentar seu desempenho na força explosiva elástica reflexa $(\boldsymbol{C J 5 s})$, demonstrando eficácia de utilização do componente elástica reflexo na fase excêntrica do movimento de salto vertical em voleibolistas com os estímulos Conexões: revista da Faculdade de Educação Física da UNICAMP, Campinas, v. 8, n. 3, p. 64-83, set./dez. 2010. ISSN: 1983-9030 
competitivos. A especificidade do treinamento é um fator a ser considerado nas respostas sobre a sensibilidade de mudanças dos jovens nessa fase de maturidade biológica.

\section{REFERENCIAS}

${ }^{1}$ SEJERSTED, O. M. et al. Muscle performance--fatigue, recovery and trainability. Acta Physiologica Scandinavica, v.162, p. 181-182, 1998.

${ }^{2}$ MALINA, R. M.; BOUCHARD, C.; BAR-OR, O. Crescimento, maturação, e atividade física. São Paulo: Phorte, 2009.

${ }^{3}$ BLIMKIE, C. J. R.; SALE, D. G. Strength development and trainability during childhood. In: VAN PRAAGH, E. Pediatric anaerobic performance. Champaign: Human Kinetics, 1998. Chapter 9. p.193-224.

${ }^{4}$ CROIX, M. S. Advances in pediatric strength assessment: changing our perspective on strength development. Journal of Sports Science and Medicine, v.6, p. 292-304, 2007.

${ }^{5}$ FAIGENBAUM, A. D. et al. The effects of a twice-a-week strength training program on children. Pediatric Exercise Science, v. 5, p. 339-346, 1993.

${ }^{6}$ FAIGENBAUM, A. D.; WESCOTT, W. L.; MICHELI. L. J.; The effects of strength training and detraining on children. Journal of Strength and Conditioning Research, v.10, p.109$114,1996$.

${ }^{7}$ FAIGENBAUM, A. D. et al. The effects of different resistance training protocols on muscular strength and endurance development in children. Pediatrics, v.104, p.1-7, 1999.

${ }^{8}$ FAIGENBAUM, A. D.; MILIKEN, L. A.; WESTCOTT, W. L. Maximal strength testing in healthy children. Journal Strength Conditioning Research, v.17, p.162-166, 2003.

Conexões: revista da Faculdade de Educação Física da UNICAMP, Campinas, v. 8, n. 3, p. 64-83, set./dez. 2010. ISSN: 1983-9030 
${ }^{9}$ CHRISTOU, M.et al. Effects of resistance training on the physical capacities of adolescent soccer players. Journal of Strength and Conditioning Research, v.20, p.783-791, 2006.

${ }^{10}$ INGLE, L.; SLEAP, M.; TOLFREY, K. The effect of a complex training and detraining programmer on selected strength and power variables in early pubertal boys. Journal of Sports Sciences, v.24, p. 987 - 997, 2006.KLAUSEN; SCHIBYE; RASMUSSEN, 1989.

${ }^{11}$ MIKKOLA, J. et al. Concurrent endurance and explosive type strength training improves neuromuscular and anaerobic characteristics in young distance runners. International Journal of Sports Medicine, v. 28, p. 602-611, 2007.

12 DIALLO, O.; DORE, E.; DUCHE, P.; VAN PRAAGH, E. Effects of plyometric training followed by a reduced training program me on physical performance in prepubescent soccer players. Journal Sports Medicine and Physical Fitness, v. 41, p. 342-48, 2001.

${ }^{13}$ KOTZAMANIDIS, C. Effect of plyometric training on running performance and vertical jumping in prepubertal boys. Journal of Strength and Conditioning Research, v. 20, 441445, 2006.

${ }^{14}$ MATAVULJ, D. et al. Effects of plyometric training on jumping performance in junior basketball players. Journal of Sports Medicine and Physical Fitness, v. 41, p.159-164, 2001.

${ }^{15}$ HÄKKINEN, K.; KESKINEN, K. L. Muscle cross sectional area and voluntary force production characteristics in elite strength and endurance trained athletes and sprinters. European Journal of Applied Physiology, v. 59, p. 215-220, 1989.

${ }^{16}$ LILLEGARD, W. A. et al. Efficacy strength training in prepubescent males female: effects of gender and maturity. Pediatric Rehabilitee, v.1, p.147-157, 1997.

Conexões: revista da Faculdade de Educação Física da UNICAMP, Campinas, v. 8, n. 3, p. 64-83, set./dez. 2010. ISSN: 1983-9030 
${ }^{17}$ MERO, A.; JAKKOLA, L.; KOMI, P. V. Serum hormones and physiological performance capacity in young boys athletes during a 1 years training period, European Journal of Applied Physiology, v. 60, p.32-37, 1990.

${ }^{18}$ RAMSAY, J. A. et al. Strength effects in prepubescent boys. Medicine and Science in Sports and Exercise, v.14, p.134-143, 1990.

${ }^{19}$ BLIMKIE, C. J. Resistance during training pre-and early puberty: efficacy, trainability, mechanism, and persistence. Canadian Journal Sport Science, v.17, p. 264-279, 1992.

${ }^{20}$ FRISANCHO, A. R. Anthropometric standards for the assessment of growth and nutritional status. Ann Arbor: University Michigan Press, 1990.

${ }^{21}$ TANNER, J. M. Growth at adolescence, with a general consideration of the effects of hereditary and environmental factors upon growth and maturation from birth to maturity. $2^{\text {nd }}$ ed. Oxford: Blackwell, 1962.

${ }^{22}$ BOSCO, C.et al. Effetto della vibrazione su forza esplosiva, resistenza alla forza veloce e flessibilità muscolare. Medicina Dello Sport, v. 54, p. 287-293, 2001.

${ }^{23}$ KOMI, P. V.; BOSCO, C. Utilization of stored elastic energy in leg extensor muscles by men. Medicine and Science in Sport and Exercise, v.10, n. 14, p. 261-265, 1978.

${ }^{24}$ VAN PRAAGH, E.; DORÉ, E. Short-term muscle power during growth and maturation. Sports Medicine, v. 32, p.701-28, 2002.

${ }^{25}$ PAASUKE, M.; ERELINE, J.; GPEYEVA, H. Twitch contraction properties of plantar flexor muscles in pre and pos-puberal boys and men. European Journal Applied Physiology, v.82, p.459-464, 2000.

${ }^{26}$ WOOD, L. E. et al. Elbow flexion and extension strength relative to body or muscle size in children. Medicine and Science in Sports Exercise, v.36, p. 1977-1984, 2004.

Conexões: revista da Faculdade de Educação Física da UNICAMP, Campinas, v. 8, n. 3, p. 64-83, set./dez. 2010. ISSN: 1983-9030 
${ }^{27}$ DEIGHAN, M. et al.Measurement of maximal muscle cross-sectional area of the elbow extensors and flexors in children, teenagers and adults. Journal of Sports Sciences, v. 24, p.543-546, 2006.

${ }^{28}$ FAIGENBAUM, A. D.et al. Effects of a short-term plyometric and resistance training program on fitness performance in boys age 12 to 15 years. Journal of Sports Science and Medicine, v. 6, p. 519-525, 2007.

${ }^{29}$ GABRIEL, D. A.; KAMEN, G.; FROSN, G. Neural Adaptations to Resistive Exercise: Mechanisms and Recommendations for Training Practices. Sports Medicine, v. 36, p.133$149,2006$.

${ }^{30}$ BOJSEN-MØLLER, J. et al. Muscle performance during maximal isometric and dynamic contractions is influenced by the stiffness of the tendinous structures. Journal of Applied Physiology, v. 99, p. 2477, 2005.

${ }^{31}$ PETERSON, M. D.; ALVAR, B. A.; RHEA, M. R. The contribution of maximal force production to explosive movement among young collegiate athletes. Journal of Strength and Conditioning Research, v. 20, p.867-873, 2006.

${ }^{32}$ COUTTS, A. J.; MURPHY, A. J.; DASCOMBE, B. J. Effect of direct supervision of a strength coach on measures of muscular strength and power in young rugby league players. Journal of Strength and Conditioning Research, v.18, p.316-323, 2004.

${ }^{33}$ GOROSTIAGA, E. M. et al. Strength training effects on physical performance and serum hormones in young soccer players. European Journal of Applied Physiology, v. 93, p. 507, 2005.

${ }^{34}$ NUZZO, J. L. et al. Relationship between countermovement jump performance and multipoint isometric and dynamic tests of strength. Journal of Strength and Conditioning Research, v. 22, p. 699-707, 2008.

Conexões: revista da Faculdade de Educação Física da UNICAMP, Campinas, v. 8, n. 3, p. 64-83, set./dez. 2010. ISSN: 1983-9030 
${ }^{35} \mathrm{KUBO}, \mathrm{K}$. et al. Effects of plyometric and weight training on muscle-tendon complex and jump performance. Medicine and Science in Sports Exercise, v. 39, p. 1801-1810, 2007.

${ }^{36}$ SANTOS, E. J.; JANEIRA, M. A. Effects of complex training on explosive strength in adolescent male basketball players. Journal of Strength and Conditioning Research, v. 22, p. 903-9, 2008.

${ }^{37}$ STANGANELLI, L. C. et al. Adaptations on jump capacity in Brazilian volleyball players prior to the under-19 World Championship. Journal of Strength and Conditioning Research, v. 22, n. 3, p. 741-9, 2008. 